\title{
Detection and Localization of Transient Sources: Comparative Study of Complex-Lag Distribution Concept Versus Wavelets and Spectrogram-Based Methods
}

\author{
Bertrand Gottin, ${ }^{1}$ Cornel Ioana, ${ }^{1}$ Jocelyn Chanussot, ${ }^{1}$ Guy D’Urso, ${ }^{2}$ and Thierry Espilit ${ }^{3}$ \\ ${ }^{1}$ GIPSA-Lab, Grenoble INP, Domaine Universitaire Saint-Martin d'Heres, 38402 Saint-Martin d'Heres, France \\ ${ }^{2}$ Research and Development of EDF, 78401 Chatou, France \\ ${ }^{3}$ Research and Development of EDF, 77250 Moret sur Loing, France
}

Correspondence should be addressed to Bertrand Gottin, bertrand.gottin@gipsa-lab.grenoble-inp.fr

Received 11 June 2009; Accepted 25 September 2009

Recommended by A. Enis Cetin

The detection and localization of transient signals is nowadays a typical point of interest when we consider the multitude of existing transient sources, such as electrical and mechanical systems, underwater environments, audio domain, seismic data, and so forth. In such fields, transients carry out a lot of information. They can correspond to a large amount of phenomena issued from the studied problem and important to analyze (anomalies and perturbations, natural sources, environmental singularities, ...). They usually occur randomly as brief and sudden signals, such as partial discharges in electrical cables and transformers tanks. Therefore, motivated by advanced and accurate analysis, efficient tools of transients detection and localization are of great utility. Higher order statistics, wavelets and spectrogram distributions are well known methods which proved their efficiency to detect and localize transients independently to one another. However, in the case of a signal composed by several transients physically related and with important energy gap between them, the tools previously mentioned could not detect efficiently all the transients of the whole signal. Recently, the generalized complex time distribution concept has been introduced. This distribution offers access to highly concentrated representation of any phase derivative order of a signal. In this paper, we use this improved phase analysis tool to define a new concept to detect and localize dependant transients taking regard to the phase break they cause and not their amplitude. ROC curves are calculated to analyze and compare the performances of the proposed methods.

Copyright () 2009 Bertrand Gottin et al. This is an open access article distributed under the Creative Commons Attribution License, which permits unrestricted use, distribution, and reproduction in any medium, provided the original work is properly cited.

\section{Introduction}

Transient signals can be globally defined as impulsive or very short duration signals often with oscillations. These signals exist in many different applications and systems from underwater acoustic [1] with opportunity sources to audio signals with sound attacks [2] or electrical systems with partial discharges and faults in cables [3]. The main processing done on this type of signal aims to detect them and localize their source in multisensor configuration. One issue point concerning transient signals is that they are typically defined on very few samples and are difficult to modelize by asymptotic approach. Consequently, their characterization remains a very challenging goal of increasing interest. Until now, several signal processing tools such as Higher-Order
Statistics (HOS), Wavelets (WVT) coefficients, and wellknown Time-Frequency analysis by Spectrogram are widely used to perform the goals of detection and localization. The HOS are very suitable to detect transients drowned in additive white Gaussian noise. The HOS give high value statistics for non-Gaussian components as transients, whereas all Gaussian parts of the signal have higher-order statistics very closed to zero $[4,5]$. Wavelet coefficients and spectrogram are based on energy criteria and give high energetical value signatures where transient components occur $[6,7]$. One of the common points of these approaches is the use of energy coefficients to prove the transients detection. Alternatively, the transients could be detected via the changes of signal instantaneous phase. Hence, one promising approach consists of analyzing the instantaneous 
phase derivatives of the studied signal and to detect the transitions as the signal parts which provide significant values of phase derivatives at several orders. Recently, for phase derivatives analysis, the time-frequency distribution based on complex lag arguments has been introduced $[8,9]$. This distribution is able to reduce inner interferences terms which appear when studying nonlinear TF components. It also offers access to an instantaneous law representation of any phase derivative order.

Unlikely to HOS, WVT, and Spectrogram, this concept of complex-lag distribution focuses directly on the phase law of the signal with no regard to its amplitude. Therefore, in a task of transients detection, when the amplitude of the transient is too low, the statistics obtained by HOS (Skewness, Kurtosis or fourth-order cumulant) as well as WVT coefficients could be too weak and not enough significant for detection. In the same way, the spectrogram distribution could miss low amplitude transients masked by other transients or components of strong amplitude. Using the complex-lag distribution, despite low amplitude signal, the phase break due to the transient remains significant and visible for detection in the "Time-Phase Derivatives" representation plane.

In this paper, we consider transient signals from a multipath configuration. This configuration is encountered in many application areas such as electrocardiograms (EKG), underwater acoustic, seismic, and so forth. A good illustrative case of such configuration signals is the one of signals from reflectometry studies using Gaussian pulse transmission in electric power cables. This reflectometry signal is composed by the emitted pulse and several other pulses due to some reflections on particular cable spots (end of cable, junction points, faults points) [10]. These reflection pulses come back to the cable reflectometry transmission point with some delay, amplitude attenuation, and phase shift.

The paper is organized as follows. In Section 2 a presentation of the complex time distribution concept is done. The capability of each method to deal with full detection and localization of transients contained in typical reflectometry signals is presented in Section 3. We conclude in Section 4.

\section{Time-Frequency Distribution Based on Complex Lag Arguments}

The concept of complex lag distributions has been introduced in [8] as a way for inner interferences reduction with respect to Wigner distribution. Recently, this concept has been generalized in order to focus on arbitrary instantaneous phase derivative of a signal [9]. Let us consider the signal defined as

$$
s(t)=A \cdot e^{j \phi(t)} .
$$

The case of $A$ depending of $t$ can also be addressed since the effect of slowly varying amplitude is "visible" on the instantaneous phase. Otherwise, after a signal normalization, we can consider $A=1$.

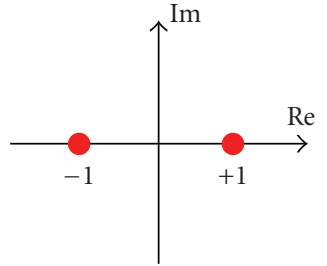

FIgURE 1: Lag coefficients taken on the real axis.

In order to better understand the concept of complex lag distribution and its generalization, applied on such a signal, let us introduce the very well-known Wigner distribution with appropriate analysis of its moment and lags definition.

2.1. The Wigner Ville Distribution. The Wigner Ville distribution of a signal $s(t)$ is by definition [11]:

$$
\mathrm{WVD}(t, \omega)=\mathfrak{F}_{\tau}[\overbrace{s\left(t+\frac{\tau}{2}\right) s^{*}\left(t-\frac{\tau}{2}\right)}^{M_{\mathrm{wv}}(t, \tau)}] .
$$

This corresponds to the Fourier transform, with respect to the lag variable $\tau$, of a higher-order moment denoted $M_{\mathrm{wv}}(t, \tau)$. As illustrated in Figure 1, this moment is calculated using two lag coefficients taken on the real axis.

For a signal defined in (1), the expression of the moment becomes

$$
M_{\mathrm{Wv}}(t, \tau)=e^{j[\phi(t+\tau / 2)-\phi(t-\tau / 2)]} .
$$

Let us express the signal phase law in terms of Taylor series expansion:

$$
\begin{aligned}
& \phi\left(t+\frac{\tau}{2}\right)=+\phi^{\prime}(t) \frac{\tau}{2^{1} 1 !}+\phi^{(2)}(t) \frac{\tau^{2}}{2^{2} 2 !}+\phi^{(3)}(t) \frac{\tau^{3}}{2^{3} 3 !}+\cdots, \\
& \phi\left(t-\frac{\tau}{2}\right)=-\phi^{\prime}(t) \frac{\tau}{2^{1} 1 !}+\phi^{(2)}(t) \frac{\tau^{2}}{2^{2} 2 !}-\phi^{(3)}(t) \frac{\tau^{3}}{2^{3} 3 !}+\cdots .
\end{aligned}
$$

Using the derivation results above, the expression (3) becomes

$$
M_{\mathrm{wV}}(t, \tau)=e^{j \phi^{\prime}(t) \tau} \times e^{j\left[\phi^{(3)}(t)\left(\tau^{3} / 2^{2} 3 !\right)+\cdots\right]} .
$$

By substituting (5) in (2), we obtain a new analytical expression (6) of the WVD defining it as an ideally concentrated representation of the Instantaneous Frequency Law (IFL), but degraded because of the convolution with a spreading factor.

$$
\operatorname{WVD}(t, \omega)=\delta\left(\omega-\phi^{\prime}(t)\right) *_{\omega} \mathfrak{F}_{\tau}\left[e^{j Q_{\mathrm{wv}}(t, \tau)}\right],
$$

where $Q_{w v}$ is the spread function defined as

$$
\begin{aligned}
& Q_{\mathrm{wv}}(t, \tau) \\
& \quad=\phi^{(3)}(t) \frac{\tau^{3}}{2^{2} 3 !}+\phi^{(5)}(t) \frac{\tau^{5}}{2^{4} 5 !}+\phi^{(7)}(t) \frac{\tau^{7}}{2^{6} 7 !} \cdots .
\end{aligned}
$$






FIGURE 2: Lag coefficients taken on the real and imaginary axis.

From this spread function expression, it is easy to understand that the concentration of the WV representation for a chirp signal (polynomial phase law of second order) will be optimal in so far as all $\phi$ 's derivates terms in $Q_{w v}$ will be equal to zero.

2.2. The Complex-Time Distribution. The Complex-Time distribution of a signal $s(t)$ is by definition [8]:

$$
\begin{aligned}
& \operatorname{CTD}(t, \omega) \\
& =\mathfrak{F}_{\tau}[\overbrace{s\left(t+\frac{\tau}{4}\right) s^{*}\left(t-\frac{\tau}{4}\right) s^{-j}\left(t+j \frac{\tau}{4}\right) s^{j}\left(t-j \frac{\tau}{4}\right)}^{M_{\mathrm{ct}}(t, \tau)}] .
\end{aligned}
$$

In the same way as WVD, this corresponds to the Fourier transform, with respect of the lag variable $\tau$, of a higher order moment denoted $M_{\mathrm{ct}}(t, \tau)$. As illustrated in Figure 2, this moment is in this case of order four and calculated using two lag coefficients taken on the real axis as well as on the imaginary axis, hence the concept of "complex-time arguments emerges".

Following the same frame of analysis described in Section 2.1 leads to a new expression of CTD defined with the same form as (6). The spread function for this distribution is [8]

$$
Q_{\mathrm{ct}}(t, \tau)=\phi^{(5)}(t) \frac{\tau^{5}}{4^{4} 5 !}+\phi^{(9)}(t) \frac{\tau^{9}}{4^{8} 9 !}+\phi^{(13)}(t) \frac{\tau^{13}}{4^{12} 13 !} \cdots .
$$

Defining a distribution using well-chosen "complex-lag" arguments $(+j$ and $-j$ on the imaginary axis) involves a significant decreasing of the spread factor. The first term of $Q_{\mathrm{ct}}(t, \tau)$ is of the fifth order. The terms of phase derivatives of order $3,7,11, \ldots$ are completely eliminated and all remaining terms are much more reduced with respect to the ones in the Wigner distribution. The Complex Time Distribution improves the concentration of the IFL representation comparing to the one obtained by Wigner Distribution (Figure 4). In the case of a nonlinear and rapidly varying TF structure, the inner interferences are strongly reduced.

2.3. The Generalized Complex-time Distribution. Recently, a generalization of the concept of CTD has been defined [9]. The starting point of this generalization procedure was

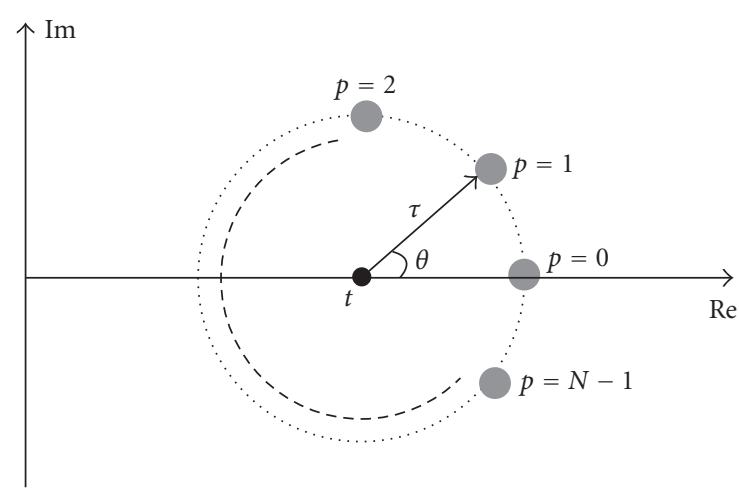

Figure 3: The complex lag coordinates.

the Cauchy's integral formula [12]. Using this theorem, it is possible to compute the $K$ th order derivative of the instantaneous phase as

$$
\phi^{(K)}(t)=\frac{K !}{2 \pi j} \oint_{\gamma} \frac{\phi(z)}{(z-t)^{K+1}} d z .
$$

This relation shows the interest of the complex time concept: the $K$ th order derivate of function $\phi$ at instant $t$ (which could correspond to one signal singularity) can be computed as the complex integral over the integration path $\gamma$ defined, in the complex plane, around this point. Applying the theory of Cauchy's integral theorem [12] and considering a circle as integration path, the expression (10) becomes [9]

$$
\phi^{(K)}(t)=\frac{K !}{2 \pi \tau^{K}} \int_{0}^{2 \pi} \phi\left(t+\tau e^{j \theta}\right) e^{-j K \theta} d \theta .
$$

As illustrated in Figure 3, the discrete version of (11) is defined for $\theta=2 \pi p / N$ and $p=0, \ldots, N-1$, where $N$ is the number of discrete values of the angle $\theta$ (expression (12)).

$$
\phi^{(K)}(t)=\frac{K !}{N \tau^{K}} \sum_{p=0}^{N-1} \phi\left(t+\tau e^{j(2 \pi p / N)}\right) e^{-j(2 \pi p K / N)}+\varepsilon
$$

where $\varepsilon$ is the discretization error.

Using the property of the unitary roots $\omega_{N, p}=e^{j 2 \pi p / N}$ and the variable change $\tau \leftarrow \sqrt[K]{\tau(K ! / N)}$, the expression (12) becomes (cf. Appendix A)

$$
\sum_{p=0}^{N-1} \phi\left(t+\omega_{N, p} \sqrt[K]{\tau \frac{K !}{N}}\right) \omega_{N, p}^{N-K}=\phi^{(K)}(t) \tau+Q(t, \tau),
$$

where $Q$ is the spread function defined as [9]:

$$
Q(t, \tau)=N \sum_{r=1}^{+\infty} \phi^{(N r+K)}(t) \frac{\tau^{N r / K+1}}{(N r+K) !}\left(\frac{K !}{N}\right)^{N r / K+1} .
$$

As indicated by (13) and (14), the sum of the phase samples defined in the complex coordinates (left side of (13)) is linear depending on $\tau$ if the $\phi$ 's derivates of orders greater than $N+K$ are 0 . In order to exploit this property we 
define the generalized complex-lag moment (GCM) of $s$ as the operation leading to (13):

$$
\begin{aligned}
\operatorname{GCM}_{N}^{K}[s](t, \tau) & =\prod_{p=0}^{N-1} s^{\omega_{N, p}^{N-K}}\left(t+\omega_{N, p} \sqrt[K]{\tau \frac{K !}{N}}\right) \\
& =e^{j \phi^{(K)}(t) \tau+j Q(t, \tau)} .
\end{aligned}
$$

The computation of GCMs implies the evaluation of signal samples at complex coordinates. This is achieved using the analytical continuation of a signal defined as [9]

$$
s(t+j m)=\int_{-\infty}^{+\infty} S(f) e^{-2 \pi m f} e^{j 2 \pi f t} d f,
$$

where $S(f)$ is the Fourier transform of signal $s$. Taking the Fourier transform of GCM with respect to $\tau$, we define the generalized complex-lag distribution (GCD):

$$
\begin{aligned}
\operatorname{GCD}_{N}^{K}[s](t, \omega) & =\mathfrak{F}_{\tau}\left[\operatorname{GCM}_{N}^{K}[s](t, \tau)\right] \\
& =\delta\left(\omega-\phi^{(K)}(t)\right) *{ }_{\omega} \mathfrak{F}_{\tau}\left[e^{j Q(t, \tau)}\right] .
\end{aligned}
$$

As stated by this definition, the $K$ th order distribution of the signal, obtained for $N$ complex-lags, highly concentrates the energy around the $K$ th order derivate of the phase law. This concentration is optimal if the $\phi$ 's derivates of orders greater than $N+K$ are 0 , exactly like in the case of chirps represented by Wigner distribution.

The general definition (17) leads to a large number of TF Representations (TFRs), part of them well known in literature. For example, for $K=1 ; N=2$ the WVD is obtained (Section 2.1), whereas the case $K=1 ; N=$ 4 corresponds to the complex-time distribution (CTD) (Section 2.2). In [5], we have shown that increasing the number of complex lags leads to an attenuation of inner interferences due to the time-frequency nonlinearity. This is illustrated by the example in Figure 4 for the following test signal having a transient-behavior TF structure:

$$
s_{1}(t)=e^{j(3 \cos (\pi t)+\cos (3 \pi t)+(2 / 3) \cos (6 \pi t)+(1 / 3) \cos (9 \pi t))} .
$$

We remark the better concentration of time-frequency energy in the case of $\mathrm{GCD}_{6}^{1}$ than in the case of the other TFRs. This is analytically proved by the spread function expression (14) and illustrated by the example in Figure 4.

The next example (Figure 5) points out the derivability property of GCD. The signal $s_{2}$ used in this example is a train of three frequency modulations (FM) corrupted by some additive noise $(\mathrm{SNR}=35 \mathrm{~dB})$. The three FM have short duration (two linear FM $s_{2-1}$ and $s_{2-3}$ in phase opposition defined on 128 samples and one parabolic FM $s_{2-2}$ defined on 64 samples) compared to the analysis time frame (1510 samples). Their analytical expressions are given by:

$$
\begin{gathered}
s_{2-1}(t)=e^{j \phi_{2-1}(t)}=e^{j\left(c_{0}+c_{1} t+c_{2} t^{2}\right)}, \\
s_{2-2}(t)=e^{j \phi_{2-2}(t)}=e^{j\left(d_{0}+d_{1} t+d_{2} t^{2}+d_{3} t^{3}\right)}, \\
s_{2-3}(t)=s_{2-1}^{*}(t)=e^{j \phi_{2-3}(t)}=e^{-j\left(c_{0}+c_{1} t+c_{2} t^{2}\right)} .
\end{gathered}
$$

TABLE 1: Expressions of the first and second phase derivatives.

\begin{tabular}{lc}
\hline First $\phi$ 's derivative & Second $\phi$ 's derivative \\
\hline$\phi_{2-1}^{\prime}(t)=c_{1}+2 c_{2} t$ & $\phi_{2-1}^{(2)}(t)=2 c_{2}$ \\
$\phi_{2-2}^{\prime}(t)=d_{1}+2 d_{2} t+3 d_{3} t^{2}$ & $\phi_{2-2}^{(2)}(t)=2 d_{2}+6 d_{3} t$ \\
$\phi_{2-3}^{\prime}(t)=-c_{1}-2 c_{2} t$ & $\phi_{2-3}^{(2)}(t)=-2 c_{2}$ \\
\hline
\end{tabular}

Such signal could correspond to a received signal from two different radars using linear and parabolic FM waveforms, respectively (Figure 5(a)). The derivability capability of GCD is used here to enable the characterization of transient natures which would be more difficult using just timefrequency representation because of confusing TF signatures. As shown in Figure 5(b) and expressed in Table 1, the two linear FM have their well-expected TF structure whereas the transient parabolic FM looks like a chirp in the TF plane. This is because of the short duration effect on the large frame of analysis. We can observe that the linear and parabolic shapes of the FM are not easily distinguishable. The parabolic shape appears to be linear. Without a priori knowledge about the signal, the TF representation alone leads to consider three transients of chirp nature which is actually wrong. To point out the true nature of the parabolic FM, the $\mathrm{GCD}_{6}^{2}$ is used. As analytically proved by the equations in Table 1 , considering the second-order phase derivative allows to stationarize the linear FM and gives a linear signature for the parabolic FM (Figure 5(c)).

The TF rate representation plane avoids the previous confusion.

\section{Transients Detection by Wavelets and Higher-Order Statistics, Spectrogram and Complex-Lag Distribution}

In this section, we compare the phase derivative capabilities of GCD, in the context of transients detection, with the wavelet transform and time-frequency based methods. The signal used in this section comes from a reflectometry study using a Gaussian pulse emission in electric cables. Reflectometry is used to analyze and control anomalies in electric cables. A signal is emitted at one extremity of the cable and during its propagation when there is a fault at some point of the cable, a part of the emitted signal, amplitude is reflected due to the impedance discontinuity at this point. The reflected part of the signal comes back to the cable extremity point. The aim of the reflectometry study is to detect and localize the faults by analyzing the signal at the emission point after its propagation and reflections. In our case, a Gaussian pulse is used as emission signal at the entry point of a cable network. The cable network corresponds here to two different cables separated by a junction (Figure 6). During the propagation in the line "cable 1 -junction-cable 2", the pulse is reflected at junction points P2 and P5, faults points if there are some, and end of line point P6. As illustrated in Figure 7, the overall analyzed signal is composed by the original emitted pulse and the reflected pulses affected by some delays, amplitude attenuation, and 


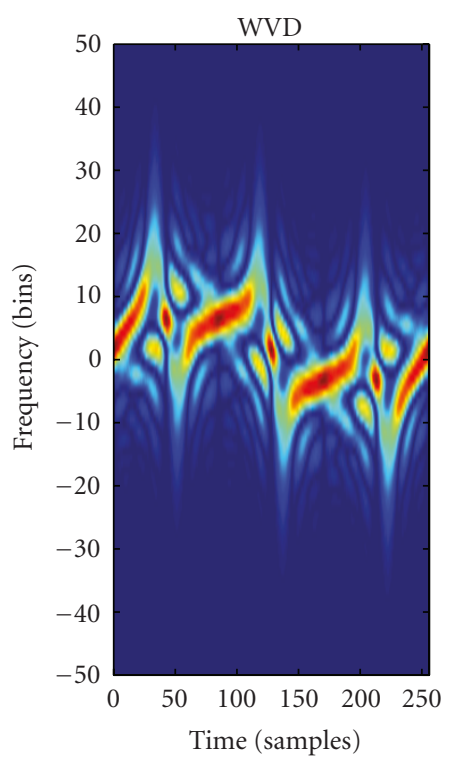

(a)

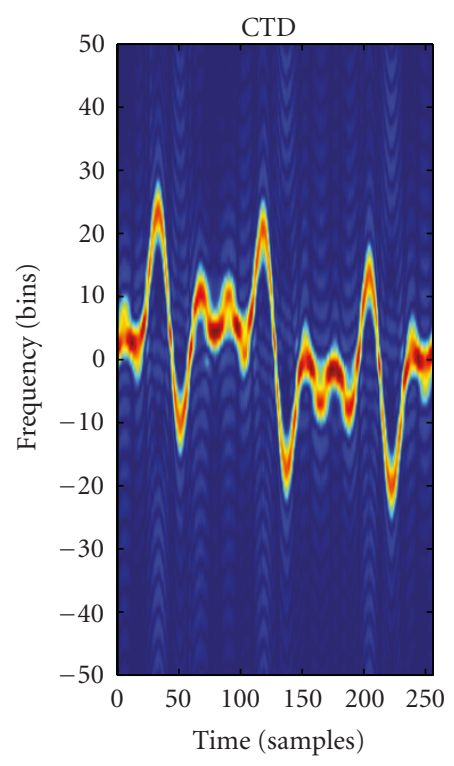

(b)

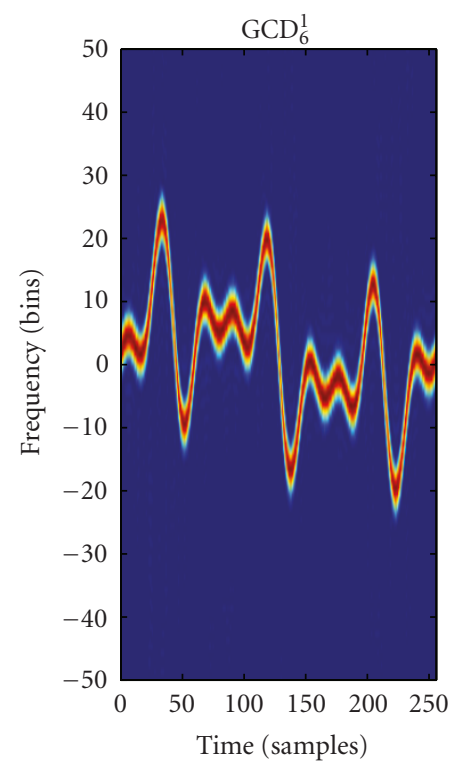

(c)

FIGURE 4: Inner interferences reduction property of GCD.

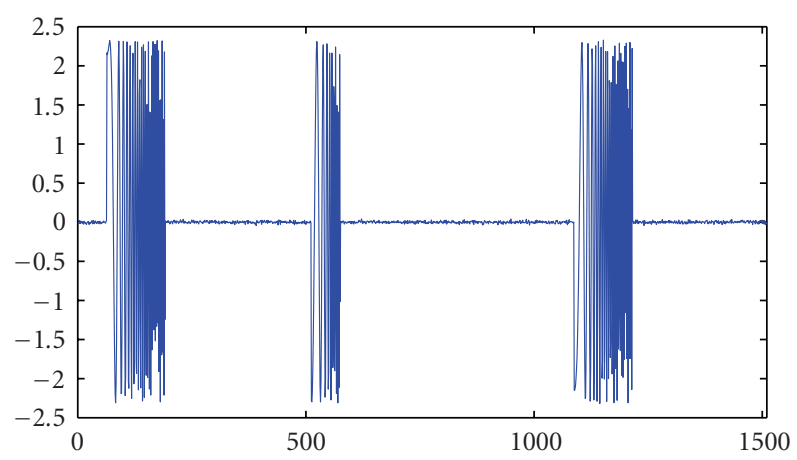

(a) Real part of signal

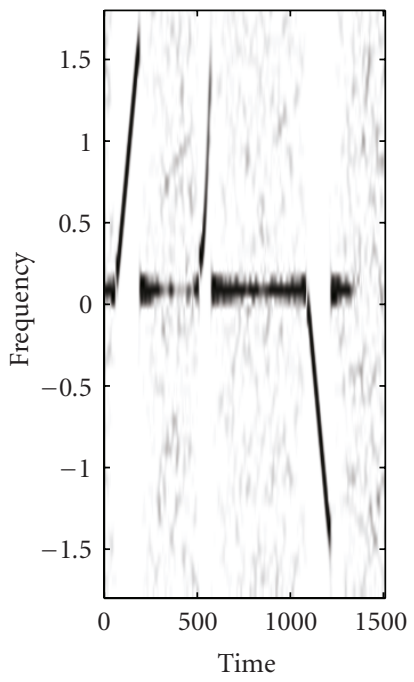

(b) $\mathrm{GCD}_{2}^{1}$

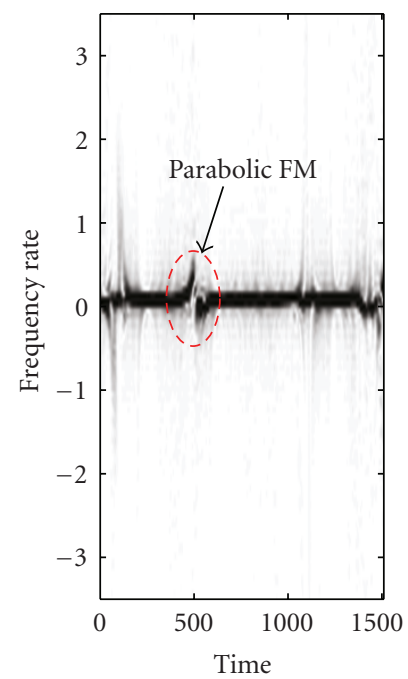

(c) $\mathrm{GCD}_{6}^{2}$

FIGURE 5: (a) Signal $s_{2}$ composed of two linear FM, one parabolic FM and additive noise (SNR = $\left.35 \mathrm{~dB}\right)$; (b) $\mathrm{GCD}_{2}^{1}$ of $s_{2}$; (c) GCD (c) $_{6}^{2}$. 


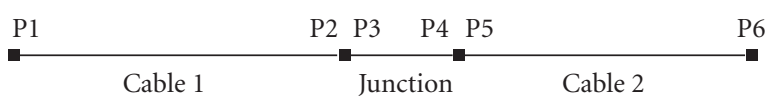

FIGURE 6: Configuration scheme with two cables separated by one junction.

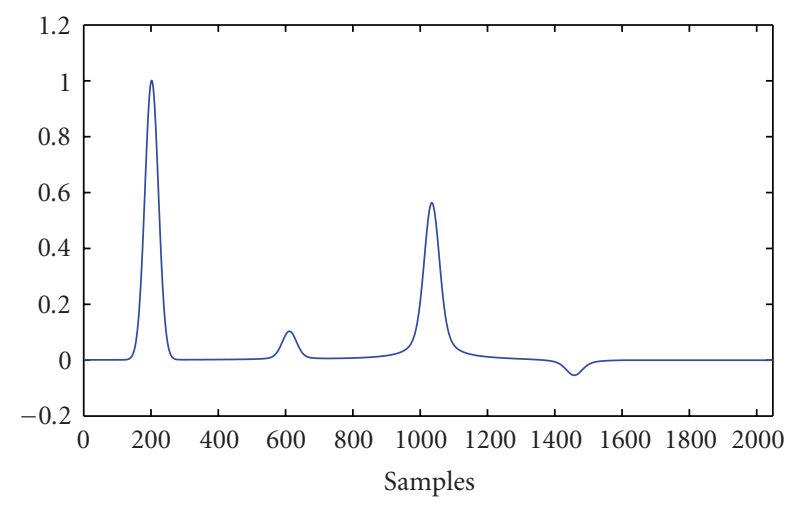

FIgURE 7: Total signal obtained at the cable entry point P1.

phase shifts. Figure 8 explains more precisely how the different transients composing the signal are matched to the propagation of the emitted pulse in the cable network physical system.

3.1. Method of Higher-Order Statistics and Wavelets. Higherorder statistics measure the non-Gaussianity of a component. Therefore, for a signal composed of transients with additive white Gaussian noise, HOS allow to detect them by giving high values for transient components that are overGaussian and putting to zero the rest of the signal corresponding with Gaussian noise. The fourth-order cumulant and the Kurtosis (normalized version of 4 th-order cumulant) are the HOS usually considered in time or in frequency. The 4th-order cumulant and time Kurtosis use the signal in time domain and are obtained via estimators calculated from the signal samples and affected by bias and variance [4]. Their frequency versions use estimators calculated with the signal samples in frequency domain, that is, $X(\nu)=\operatorname{TF}[x(t)]$.

The wavelet transform is widely used for transients detection. Using Discrete Wavelet Transform, the signal is decomposed in a time-scale plane segmented in blocks and representing a wavelets basis. Each block is a basis vector and corresponds to the main wavelet affected by some delay and scale factor. The representation obtained by DWT is formed by wavelet coefficients which are the projections of the signal on each basis vector. DWT proves its efficiency in transients detection in so far as wavelets signals are usually very similar to transient signals. Consequently, the DWT distribution gives higher inner products between transients and wavelet basis vectors. Transients are then detected and located where higher energetical wavelet coefficients are obtained in the distribution.

In 1996, an efficient method blending the advantages of both HOS and wavelets was proposed to detect and localize transient signals with improved performances $[5,13$,
14]. This method is based on the adapted segmentation of the time-frequency plane by Malvar wavelets. The criteria used to fit the wavelets for the TF plane segmentation are based on HOS, especially the 4th-order cumulant. The final detection curve corresponds to this 4 th-order cumulant calculated at each time-delay regions resulted from the final wavelet distribution optimally segmented in terms of Gaussian and non-Gaussian signal components. This curve will be then normalized for the performance analysis by ROC curves.

Figures 9 and 10 show the good performances of this method to detect efficiently transient components embedded in white Gaussian noise with very low SNR. The two strongest transients of the signal noised at $\mathrm{SNR}=-8 \mathrm{db}$ are as well detected as in the case SNR $=30 \mathrm{db}$. However, the limitation of this method, in terms of simultaneous detection of all the transitions contained in the signal, is illustrated as well. With a signal to noise ratio of $30 \mathrm{db}$, the four transients of the signal are all well visible but, as well as the case $\mathrm{SNR}=-8 \mathrm{db}$ for which the transients are highly drowned in the noise, they cannot be detected in the same time in an efficient way. This is because of the amplitude difference between the two highest pulses (from emission and end of line reflection in the cable network) and the two lowest ones. From Figure 8, we know that the two lowest pulses result from the presence of a junction separating the two cables. As shown in Figure 9, the detection curve gives for the two low pulses HOS quasi equal to zero relatively with the highest HOS from the other pulses. Consequently, such a detection result leads to consider that we have only the emitted pulse and its reflection at the end of the line but with no presence of a junction. The physical system is therefore badly interpreted. As illustrated in Figure 11, in terms of ROC curves (cf. Appendix B), for a threshold equal to zero the detection and false alarm probabilities (Pd and Pfa) are equal to one, but for a threshold higher than zero of just one calculation step equal to 0.01 , the $\mathrm{Pd}$ cannot be more than $50 \%$. However, the Pfa is always quasi equal to zero, even when SNR is $-8 \mathrm{db}$.

3.2. Method of Spectrogram. In order to eliminate the limitations of the Fourier Transform when analyzing nonstationary signals, the idea is to consider the signal as locally stationary on an adapted time frame. The principle is to make the Fourier analysis of successive time blocks of the signal weighted by a time frame (Uniform, Hanning, Hamming ...). This is equivalent to express the signal by a set of basis functions localized both in time and frequency [11]:

$$
\begin{aligned}
\operatorname{STFT}_{x}^{(h)}(t, f) & =\int_{\mathbf{R}} x(\theta) h_{t, f}^{*}(\theta) d \theta \\
& =\int_{\mathbf{R}} x(\theta) h^{*}(\theta-t) e^{-j 2 \pi f \theta} d \theta .
\end{aligned}
$$

The above expression corresponds to the inner product between signal $x(t)$ and the basis functions $h_{t, f}(\theta)=h(\theta-t) e^{-j 2 \pi f \theta}$. The representation resulting from relation (20) is named the Short Time Fourier Transform-STFT. According to its definition, the STFT 


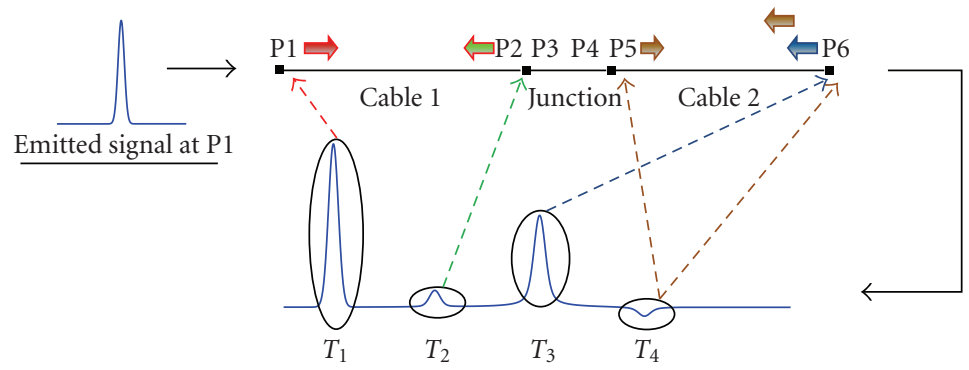

Total signal obtained at $\mathrm{P} 1$ after propagation and reflections

FIGURE 8: $T_{1}=$ emitted pulse at P1; $T_{2}=$ pulse resulting from the reflection at junction point $\mathrm{P} 2($ cable $1 /$ junction $)$ of $T_{1} ; T_{3}=$ pulse resulting from the reflection at end of line point P6 of $T_{1}-T_{2} ; T_{4}=$ pulse resulting from the reflection at $\mathrm{P} 6$ of the part of $T_{3}$ coming from its reflection at junction point P5 (cable2/junction).

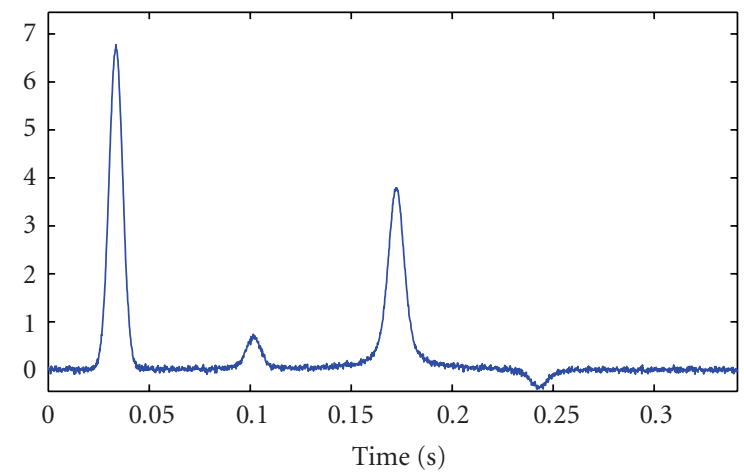

(a) Analyzed signal

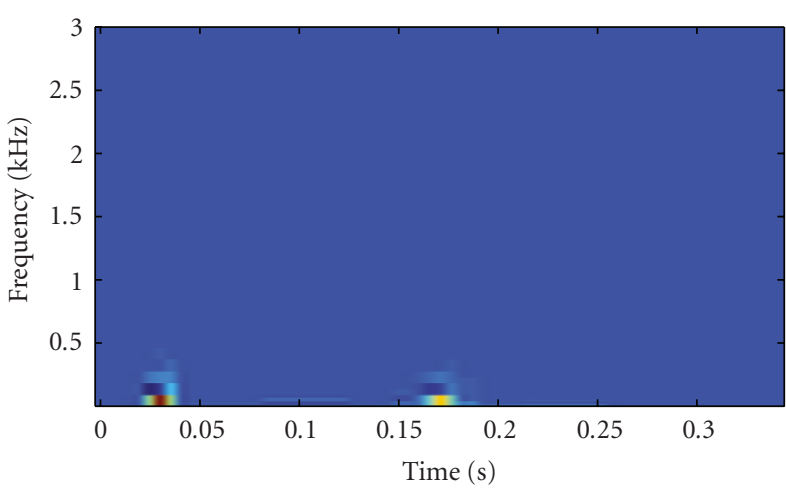

(c) Malvar wavelets decomposition

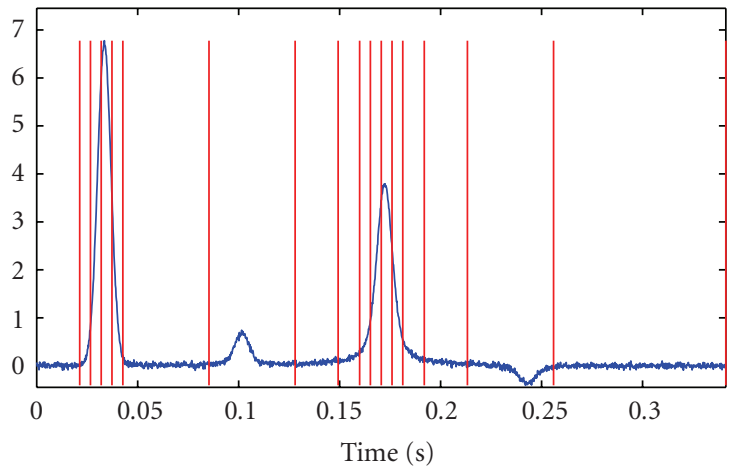

(b) Adapted segmentation

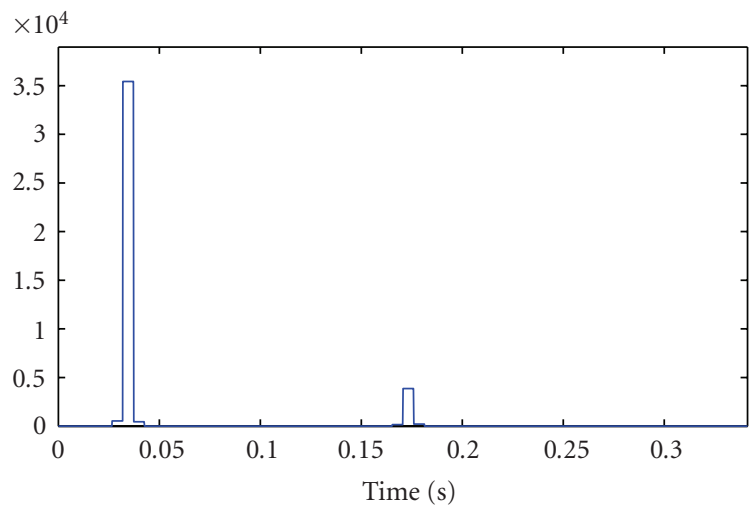

(d) Detection curve

FIGURE 9: Detection of transient components by adapted wavelet analysis; SNR $=30 \mathrm{db}$.

of a signal is a complex values representation. For this reason, its square modulus is generally represented and used as a traditional Time-Frequency (TF) distribution. This distribution is named the Spectrogram [11]. The spectrogram as well as the STFT considers actually the whole nonstationary signal as a succession of short quasi-stationary signals defined on the time domain of the weighting frame $h(u)$. These TF representations are limited by the Heisenberg uncertainty principle [11]:

$$
\begin{gathered}
\Delta t \cdot \Delta f \geq \frac{1}{4 \pi}, \\
(\Delta t)^{2}=\frac{\int_{-\infty}^{+\infty} t^{2}|x(t)|^{2} d t}{\int_{-\infty}^{+\infty}|x(t)|^{2} d t}, \quad(\Delta f)^{2}=\frac{\int_{-\infty}^{+\infty} f^{2}|X(f)|^{2} d f}{\int_{-\infty}^{+\infty}|X(f)|^{2} d f},
\end{gathered}
$$




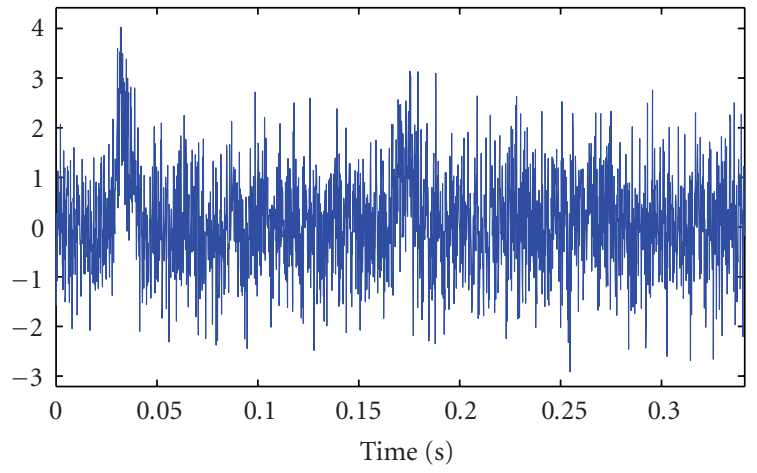

(a) Analyzed signal

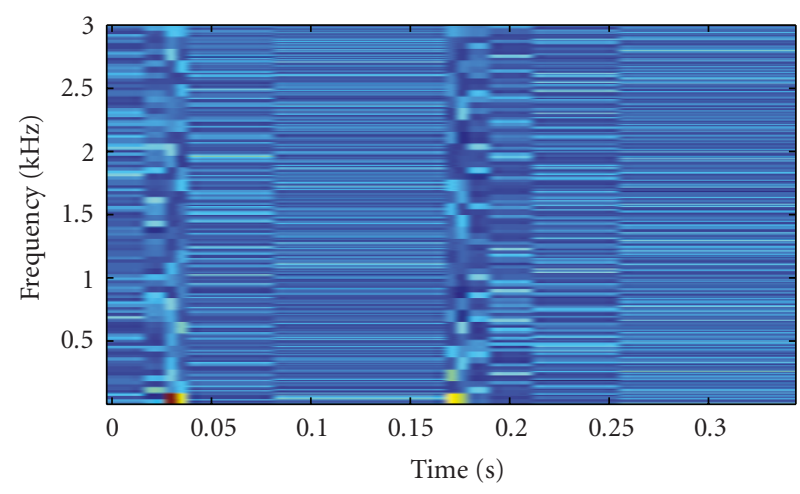

(c) Malvar wavelets decomposition

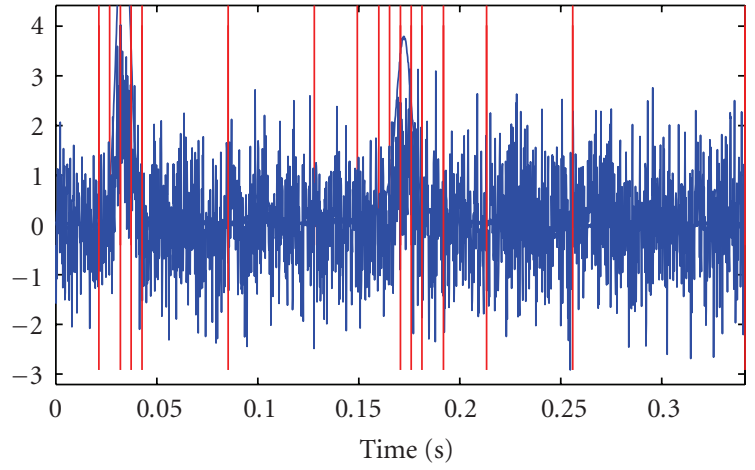

(b) Adapted segmentation

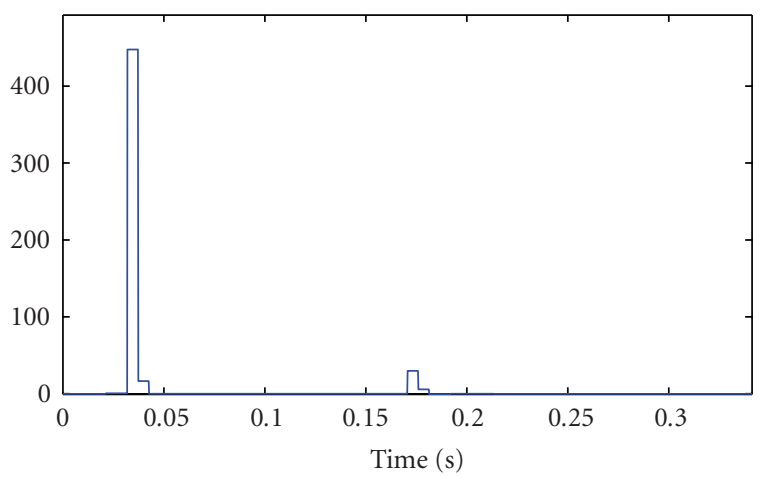

(d) Detection curve

FIGURE 10: Detection of transient components by adapted wavelet analysis; SNR $=-8 \mathrm{db}$.

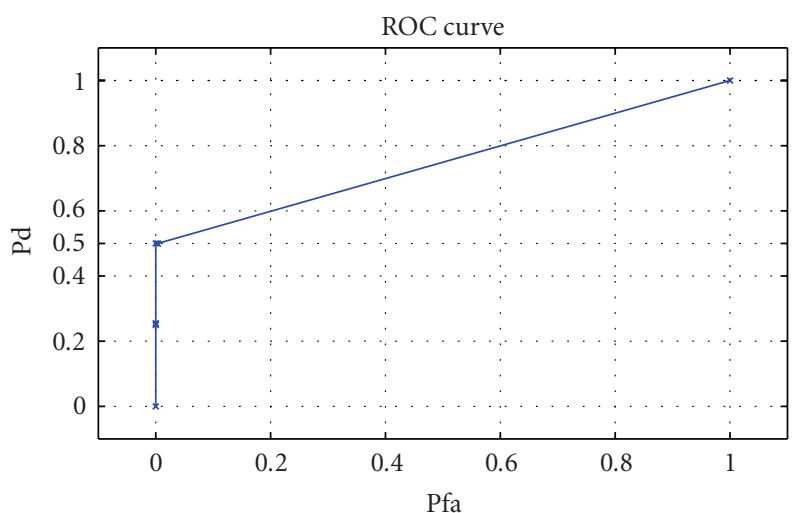

FIGURE 11: ROC curve for detection based on Malvar wavelet adapted segmentation.

where $\Delta t$ and $\Delta f$ are respectively the distribution resolution in time and frequency. This uncertainty principle means that the distribution cannot have in the same time good resolution in time and frequency. These two parameters are antagonistic and there is always a trade-off to do between time and frequency resolutions.

The methodology of detection based on spectrogram consists in calculating the spectrogram distribution of the reflectometry signal (Figure 7). Our study focuses on detection and localization of transient signals which are, by definition, short in time and large band in frequency. The spectrogram is consequently calculated using a very short frame $h(u)$ in order to have a good resolution in time resulting in a limited resolution in frequency which is not actually problematic (Figure 12). A good resolution in time is moreover suitable and necessary in so far as two transient components of the signal can be very close. The final detection curve $\mathrm{DC}_{\text {Spectro }}(t)$ corresponds to the curve of maxima of each column of the obtained distribution. This curve will be then normalized for the performance analysis by ROC curves. As illustrated in Figure 12(a), the spectrogram of the reflectometry signal gives high energetical values where transients occur, and we can note that the energy signatures of the two small energy transients (samples 600 and 1450) are not visible. Thus, for SNR $=30 \mathrm{db}$ (the signal is the same as Figure 9(a)) the detection curve represented in Figure 12(b) shows that the two strongest pulses are well detected whereas the two lowest ones are not significantly detected. Only the low transient at sample 600 gives a very weak detection signature. As well as in Section 3.1, the two low pulses, associated with the presence of a junction in the system, have an amplitude much weaker than the other pulses. Their energy is consequently masked in the distribution by the higher energy of the other transients.

This detection based on spectrogram, as well as the adapted wavelets analysis (Section 3.1), has the advantage to 


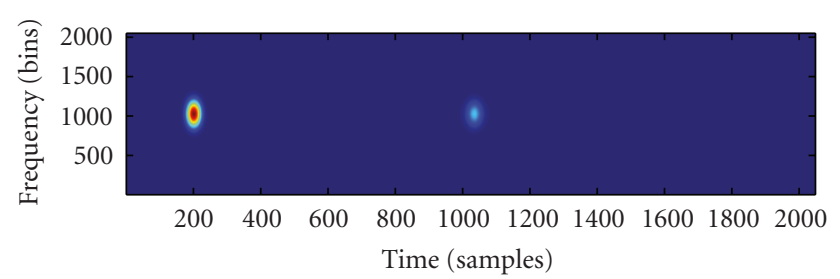

(a)

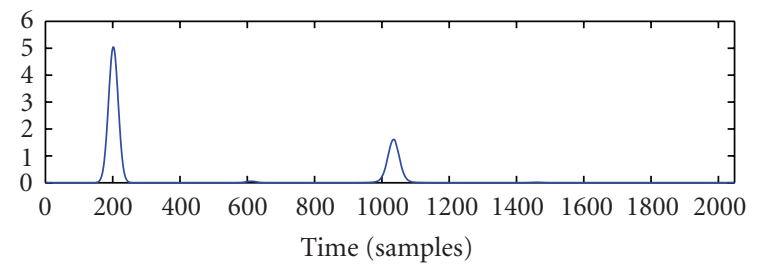

(b)

Figure 12: (a) Spectrogram of the reflectometry signal noised at $\mathrm{SNR}=30 \mathrm{db}$; (b) associated detection curve.

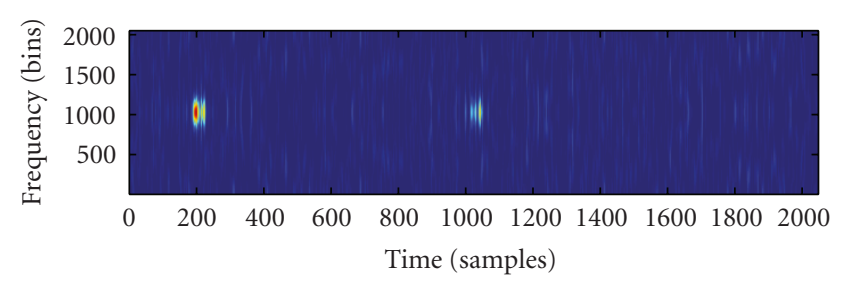

(a)



(b)

Figure 13: (a) Spectrogram of the reflectometry signal noised at $\mathrm{SNR}=-8 \mathrm{db}$; (b) associated detection curve.

be robust to noise. For SNR $=-8 \mathrm{db}$, the signal in time (the same as Figure 10(a)) is highly corrupted and the transients contained in the signal are strongly drowned in the noise. As illustrated in Figure 13(a), the distribution obtained by spectrogram still gives well visible high energetical signatures for the two strongest pulses. The energy of the two low pulses remains masked in the distribution and the noise is spread on the whole TF plane. The noisy detection curve represented in Figure 13(b) leads to a good detection of the two main transients, whereas the two weak pulses are totally drowned in the noise. This second method has the same efficiency limitations as the one presented in Section 3.1 in terms of simultaneous detection of all the transitions contained in the signal. As illustrated in Figure 14, in terms of ROC curves (cf. Appendix B), for a threshold equal to zero the Pd and the $\mathrm{Pfa}$ are equal to one. For a threshold higher than zero of just one calculation step equal to 0.01 , the two main pulses and

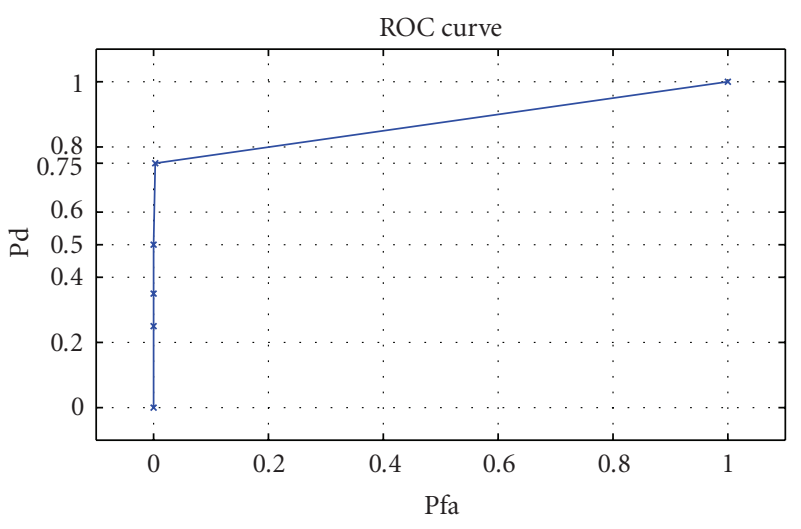

FIGURE 14: ROC curve for detection by spectrogram for SNR = $30 \mathrm{db}$.

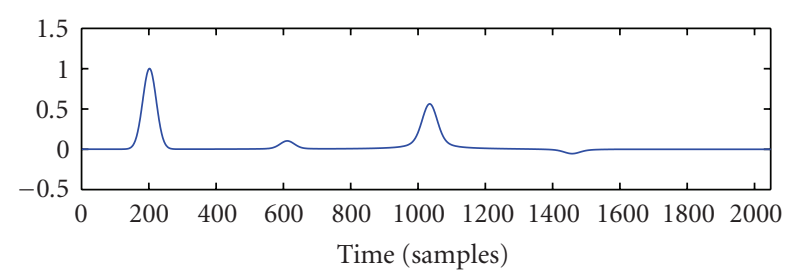

(a) Reflectometry signal $s$

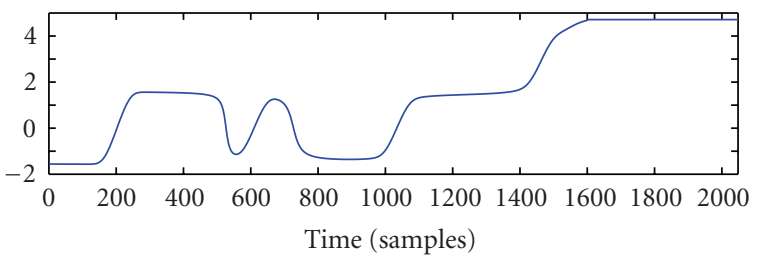

(b) instantaneous phase law

Figure 15: (a) Reflectometry signal $s$; (b) instantaneous phase law of the analytic signal associated to $s$.

the first small one are detected. The Pd is consequently of $75 \%$. However, for higher threshold the Pd cannot be more than $50 \%$ because only the two main pulses can be detected.

3.3. Method of Complex-Lag Distribution. The Generalized Complex-time Distribution (GCD) presented in Section 2.3 represents an efficient phase analysis tool. Using directly the signal samples, enables to give highly concentrated representations of any instantaneous phase derivatives, independently of the signal amplitude. Using this concept, another methodology of transients detection and localization can be defined. This methodology is based on the analysis of the signal instantaneous phase. It allows to detect efficiently and in the same time one or several transients contained in a signal. The transients are detected via the phase discontinuity they cause in the instantaneous phase and consequently with no regard to their amplitude.

In this section, the explained methodology is applied on the same reflectometry signal as before. Let us analyze the theoretical phase law of this signal. 


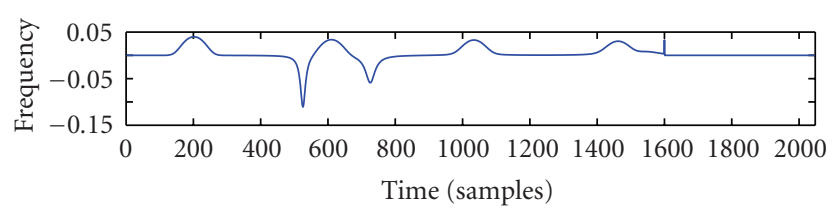

(a)

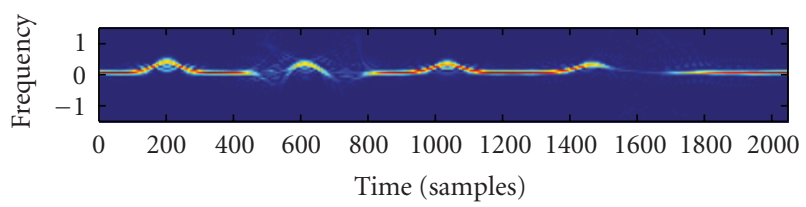

(b)



(c)

Figure 16: (a) Theoretical instantaneous frequency law (IFL) of $s$; (b) IFL obtained by GCD for SNR = $30 \mathrm{db}$; (c) associated detection curve.

As shown in Figure 15(a), signal $s$ is composed of four transients with two of them having a very low amplitude comparing to the others. This disparity "strong amplitudeweak amplitude" between the transients of the signal makes difficult the simultaneous detection of all of them, as explained in Sections 3.1 and 3.2. The two weakest pulses do not give significant signatures and are missed in detection by classical tools (HOS, wavelets, spectrogram). This limitation in detection leads to a bad interpretation of the system which will be considered as a cable line without junction. According to Figure 15(b), analyzing the phase of the signal proves its interest in so far as the four phase breaks due to the four transients have the same importance. The analytic signal associated to the real values signal $s$ is used here.

Figure 16(a) represents the theoretical instantaneous frequency law (IFL) of $s$ obtained by first derivation of the theoretical instantaneous phase law. As illustrated in Figure 16(b), the GCD calculates, using directly the signal samples, the representation of the IFL. This representation is very well concentrated around the theoretical law to analyze. The final detection curve $\mathrm{DC}_{\mathrm{GCD}}(t)$ used in this methodology comes from the curve of positions in frequency of the maximum value of each column of the obtained distribution. As the distribution obtained by GCD is well concentrated around the IFL, the argmax curve obtained is also very close to the theoretical law. The modulus of the argmax curve defines the detection curve represented in Figure 16(c). On this curve (22), all the transients are well detected with the same importance via pulse signatures resulting from the derivation of the phase discontinuities. This curve will be normalized for the performance analysis by ROC curves.

$$
\operatorname{DC}_{\mathrm{GCD}}(t)=\left|\operatorname{Arg} \max _{f}\right| \operatorname{GCD}(t, f)|| .
$$

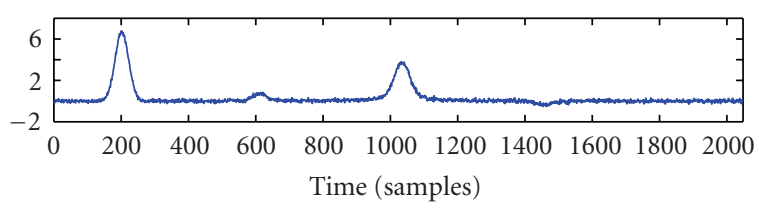

(a)

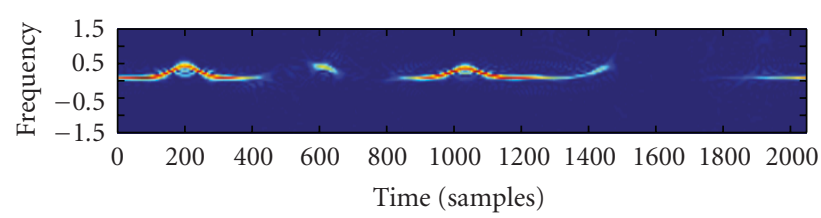

(b)

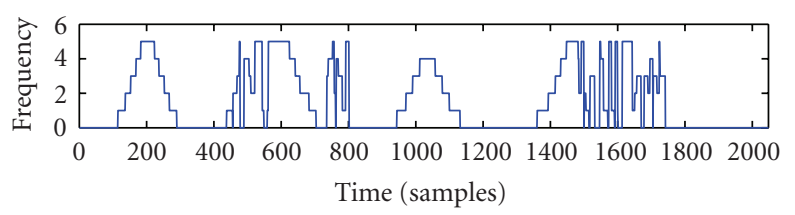

(c)

FIGURE 17: (a) Signal $s$ noised at SNR $=20 \mathrm{db}$; (b) IFL obtained by GCD; (c) associated detection curve.

Unlikely to adapted wavelets and spectrogram analysis, phase analysis is sensitive to noise. Adapted wavelets and spectrogram can detect only the two main pulses giving consequently probability of detection not more than $50 \%$ but a false alarm rate always quasi equal to zero even for very low SNR. On the opposite, detection by phase analysis allows to detect, on the same signal realization, all the transients of the signal in spite of their differences. But, as shown by Figures 17 and 18, for decreasing SNR, the sensitivity to noise of the phase analysis involves an increasing false alarm rate. However, in terms of detection performances illustrated by ROC curves Figure 19, this method allows to reach probabilities of detection which remain equal to one until very high threshold and for very low false alarm rates (cf. Appendix B).

\section{Conclusion}

In this paper, three methods for transients detection and localization have been presented in the case of an analyzed signal composed of several transients. All the transients contained in the signal result from a common physical phenomenon but are marked by strong differences between one another, in terms of difference of amplitude or phase shift. In such a context, a method by phase analysis using the tool of Generalized Complex-time Distribution proves its advantages. In terms of phase, the transient signatures by phase breaks remain all in the same way more significant than the ones obtained by consideration of energetical or statistical criteria. ROC curves illustrating and comparing the performances of the different methods lead to consider the phase analysis method as more suitable, as long as the SNR is reasonable. For very low SNR, spectrogram, wavelets, and 


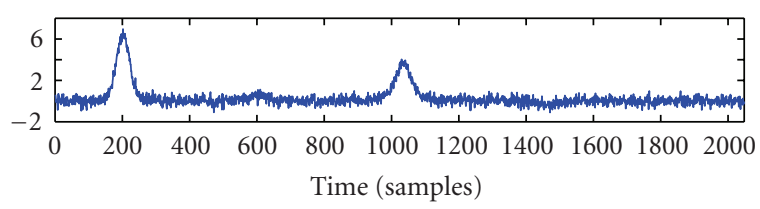

(a)

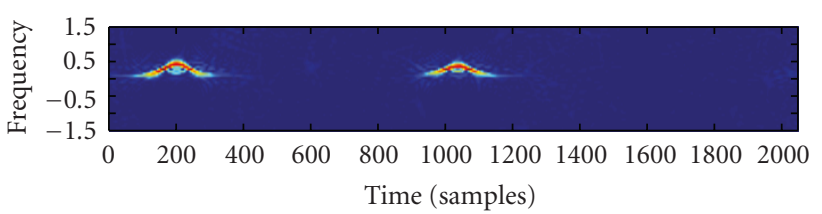

(b)

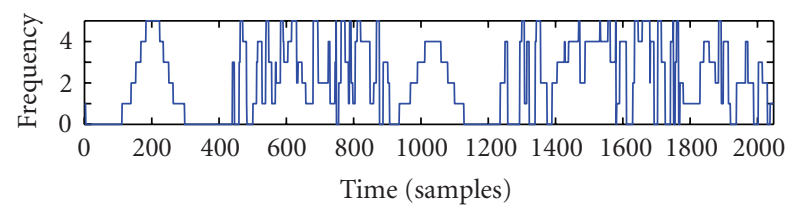

(c)

FIGURE 18: (a) Signal $s$ noised at SNR $=10 \mathrm{db}$; (b) IFL obtained by GCD; (c) associated detection curve.

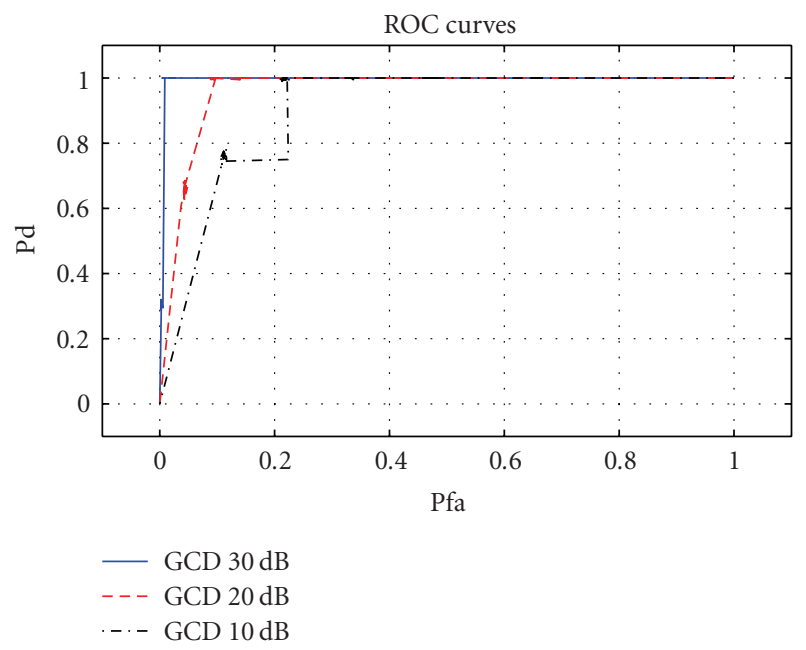

FIgURE 19: ROC curves for detection by GCD for decreasing SNR.

HOS methods remain preferable in spite of their efficiency limitation to detect all the transients of the signal.

This work about detection and localization performances represents the first step of a more complete study concerning the analysis of transients. A next step would be to characterize the transient signal. It means to be able to match a transient and its characteristics to a particular phenomenon. The Generalized Complex-time Distribution tool offers access to any derivative order of the phase law of a signal. Using this derivability property could lead to characterization via analysis of the successive phase derivates of a signal composed of several transients of different nature.

\section{Appendices}

\section{A. Derivation of the Spread Function Expression}

The final spread function expression is obtained as follows. We start by considering the discrete version of Cauchy's integral formula expressed in Section 2.3 equation (12). By using the unitary roots notation $\omega_{N, p}=e^{j 2 \pi p / N}$, this equation becomes

$$
\phi^{(K)}(t) \frac{N \tau^{K}}{K !}=\sum_{p=0}^{N-1} \phi\left(t+\omega_{N, p} \tau\right) \omega_{N, p}^{-K}+\varepsilon \frac{N \tau^{K}}{K !} .
$$

The unitary roots $\omega_{N, p}$ verify the following property:

$$
\sum_{p=0}^{N-1} \omega_{N, p}^{k}= \begin{cases}N, & \text { if } k=0(\bmod N) \\ 0, & \text { otherwise. }\end{cases}
$$

Using the Taylor series expansion of $\phi$ and the property expressed in (A.2), we obtain

$$
\begin{aligned}
\sum_{p=0}^{N-1} \phi\left(t+\omega_{N, p} \tau\right) \omega_{N, p}^{-K} & =\sum_{p=0}^{N-1}\left(\sum_{u=0}^{+\infty} \phi^{(u)}(t) \frac{\tau^{u} \omega_{N, p}^{u}}{u !}\right) \omega_{N, p}^{-K} \\
& =\sum_{u=0}^{+\infty} \overbrace{\left(\sum_{p=0}^{N-1} \omega_{N, p}^{u-K}\right)}^{\text {cf.(A.2) }} \phi^{(u)}(t) \frac{\tau^{u}}{u !} \\
& =\sum_{r=0}^{+\infty} N \phi^{(N r+K)}(t) \frac{\tau^{N r+K}}{(N r+K) !} \\
& =\phi^{(K)}(t) \frac{N \tau^{K}}{K !}+Q(t, \tau) .
\end{aligned}
$$

As a result of (A.1) and (A.3), it follows that the error term expression is

$$
Q(t, \tau)=-\varepsilon \frac{N \tau^{K}}{K !}=N \sum_{r=1}^{+\infty} \phi^{(N r+K)}(t) \frac{\tau^{N r+K}}{(N r+K) !} .
$$

Applying the variable change $\tau \leftarrow \sqrt[K]{\tau(K ! / N)}$ makes linear, comparing to variable $\tau$, the $K$ th order phase derivative term. As a result of this variable change inserted in expression (A.4), the final expression of the spread function is obtained as

$$
Q(t, \tau)=N \sum_{r=1}^{+\infty} \phi^{(N r+K)}(t) \frac{\tau^{N r / K+1}}{(N r+K) !}\left(\frac{K !}{N}\right)^{N r / K+1} .
$$

\section{B. ROC Curves Calculation}

The detection and false alarm probabilities $\mathrm{Pd}$ and $\mathrm{Pfa}$ are calculated through a statistical estimation based on a significant set of realizations of the noised signal. Let $N$ be the number of realizations, it is equal to 100 for our study. 
(i) Detection probability $P_{d}$ : each realization of the signal with additive random noise is composed of $T$ transients at known instants. On the detection curve resulted from the chosen method, we calculate $q_{i}$ corresponding to the number of good detections for each realization $i$ and for a set threshold $\eta$. The detection criteria (i.e., statistic of detection $>\eta$ ) are checked on each block of the $T$ transients, which enables a number of maximum $T$ good detections per realization. The Detection Probability is estimated as

$$
\hat{P}_{d}=\frac{\sum_{i=1}^{N} q_{i}}{T \times N} .
$$

(ii) False alarm probability $P_{f a}$ : for each realization $i$, we calculate $r_{i}$ the number of detection curve samples (transients blocks excluded), where the detection criteria are checked. Let $L$ be the length of the detection curve and $L_{\text {Block }}$ the length of each $T$ transients time domain block. The False alarm Probability for a set threshold $\eta$ is estimated as

$$
\hat{P}_{f a}=\frac{\sum_{i=1}^{N} r_{i}}{\left(L-T \times L_{\text {Block }}\right) \times N} .
$$

Each point $\left(P_{d} ; P_{f a}\right)$ of the ROC curves is obtained for a set threshold value. The final ROC curves must be calculated on a significant number of points. The threshold value $\eta$ is varying here from 0 to 1 per step of 0.01 , which involves ROC curves composed of 101 calculation points $\left(P_{d} ; P_{f a}\right)$.

\section{GCD Calculation Cost}

The GCD complexity depends on the time and frequency resolutions of the distribution, that is, the length of the signal and the range of lag $\tau$. As expressed in equation (15), the GCM is obtained by calculating, for each $\tau$, a product of $N$ complex-lagged signals. Each of these signals is obtained by the analytical continuation (16) and involves the calculation cost of an Inverse Fast Fourier Transform (IFFT matlab algorithm). Once the GCM is obtained, the GCD is obtained by Fast Fourier Transform (FFT matlab algorithm) with respect to $\tau$ at each time sample (cf. equation (17)). The GCD calculation cost is the one of multiple FFT and IFFT. It remains fast.

\section{Acknowledgments}

This work was supported by the French project PHC Pelikan. The signals of reflectometry come from a software simulating the propagation of Gaussian pulses in power cables, work realized by the Research and Development site of EDF in Paris with the participation of Guy D'Urso and Thierry Espilit.

\section{References}

[1] Z.-H. Michalopoulou, "Underwater transient signal processing: marine mammal identification, localization, and source signal deconvolution," in Proceedings of the IEEE International Conference on Acoustics, Speech, and Signal Processing (ICASSP '97), vol. 1, pp. 503-506, Munich, Germany, April 1997.

[2] C. Duxbury, M. Davies, and M. Sandler, "Separation of transient information in musical audio using multiresolution analysis techniques," in Proceedings of the COST G-6 Conference on Digital Audio Effects, Limerick, Ireland, December 2001.

[3] F. Leonard, D. Fournier, and B. Cantin, "On-line location of partial discharges in an electrical accessory of an underground power distribution network," in Proceedings of the International Conference on Insulated Power Cables (JICABLE '07), Paris, France, 2007.

[4] J. L. Lacoume, P. O. Amblard, and P. Comon, Higher Order Statistics for Signal Processing, Masson, Paris, France, 1997.

[5] P. Ravier and P.-O. Amblard, "Combining an adapted wavelet analysis with fourth-order statistics for transient detection," IEEE Transaction on Signal Processing, vol. 70, no. 2, pp. 115$128,1998$.

[6] A. Papandreou-Suppappola, Ed., Applications in TimeFrequency Signal Processing, CRC Press, Boca Raton, Fla, USA, 2003.

[7] A. Quinquis, "A few practical applications of wavelet packets," Digital Signal Processing, vol. 8, no. 1, pp. 49-60, 1998.

[8] Lj. Stanković, "Time-frequency distributions with complex argument," IEEE Transactions on Signal Processing, vol. 50, no. 3, pp. 475-486, 2002.

[9] C. Cornu, S. Stanković, C. Ioana, A. Quinquis, and Lj. Stanković, "Time-frequency distributions with generalized complex lag argument," IEEE Transactions on Signal Processing, vol. 55, no. 10, pp. 4831-4838, 2007.

[10] N. Ravot, J. Cohen, P. Chambaud, and CEA, "Method and device for analyzing electric cable networks," World Organization of Intellectual Property, WO 2008/009566 A2, January 2008.

[11] L. Cohen, Time-Frequency Analysis, Pretince-Hall, Englewood Cliffs, NJ, USA, 1995.

[12] W. Rudin, Real and Complex Analysis, McGraw Hill, Boston, Mass, USA, 1987.

[13] P. Ravier and P. O. Amblard, "Using Malvar wavelets for transient detection," in Proceedings of the IEEE-SP International Symposium on Time-Frequency and Time-Scale Analysis, pp. 229-232, Paris, France, June 1996.

[14] P. Ravier and P.-O. Amblard, "Wavelet packets and de-noising based on higher-order-statistics for transient detection," Signal Processing, vol. 81, no. 9, pp. 1909-1926, 2001. 